\title{
Seeding of Primordial Perturbations During a Decelerated Expansion
}

\author{
Yun-Song Piao \\ College of Physical Sciences, Graduate School of Chinese Academy of Sciences, YuQuan Road 19A, Beijing 100049, China
}

\begin{abstract}
A scalar field with a modified dispersion relation may seed, under certain conditions, the primordial perturbations during a decelerated expansion. In this note we examine whether and how these perturbations can be responsible for the structure formation of observable universe. We discuss relevant difficulties and possible solutions.
\end{abstract}

PACS numbers: $98.80 . \mathrm{Cq}$

\section{INTRODUCTION}

Recently, lots of observations have payed attention to the nature of primordial perturbations that gave rise to the inhomogeneities observed in the universe. The results of these measurements are consistent with an adiabatic and nearly scale invariant spectrum of primordial perturbations, as predicted by the simplest models of inflation 1]. However, due to the central role of primordial perturbations on the formation of cosmological structure, it is still very interesting and might also be desirable to explore various and possible origins of primordial perturbations.

The inflation stage is supposed to have taken place at the earlier moments of the universe [2, 3], which superluminally stretched a tiny patch to become our observable universe today, and in the meantime makes the quantum fluctuations in the horizon leave the horizon to become the primordial perturbations responsible for the formation of cosmological structure [4, 5]. This is one of remarkable successes of inflation, see also the superinflation e.g. Refs. 6, 7, 8, 9], in which the null energy condition is broken. In Ref. 6], it was firstly noticed that there is an interesting case in the generating phases of primordial perturbations, in which the scale factor and thus the wavelengths of perturbations grows very slowly but the Hubble length rapidly shrinks. The inflation can be generally regarded as an accelerated or superaccelerated stage, and so may defined as an epoch when the comoving Hubble length decreases. This length starts out very large, and then the inflation forces it to shrink enough so that the perturbations can be generated causally. In a decelerated expanding background the comoving Hubble length is increased, thus in this case it seems hardly possible to causally explain the origin of primordial perturbations. The variable speed of light [10, 11] has been considered, however, also see Ref. [12] for a reexamination. Note that it has been illustrated in Ref. [13] that the existence of adiabatic perturbations on scales much larger than the Hubble radius implies that either inflation occurred in the past, the perturbations were there as initial conditions, or causality is broken. Thus if we want to obtain the primordial perturbations in a decelerated expanding phase, we have to require that the scalar field responsible for the perturbations should have some special or modified dispersion relation. Though there have been many detailed descriptions how the required dispersion relations are obtained from the effective field theory 14], it will be still significant to examine the feasibility of this seeding mechanism matched to the observable cosmology.

The outline of this paper is as follows. In section II, we will show how the primordial perturbation may be generated during a decelerated expanding phase when the dispersion relation is modified. In this case, the perturbation spectrum is calculated in section III. We discuss relevant difficulties in matching the spectrum to observable cosmology and possible solutions. Finally, we summarize and discuss our results, and as well as give some comments on future issues.

\section{GENERATION OF SPECTRUM}

In this section we will begin with a general discussion on the generation of causal primordial perturbations. The generation of primordial perturbations requires that the perturbation modes can leave the horizon during their generation and then reenter the horizon at late time. Thus it may be convenient to define

$$
\mathcal{N} \equiv \ln \left(\frac{k_{e}}{k}\right) \equiv\left(\frac{a_{e} h_{e}}{a h}\right)
$$

which measures the efolding number of mode with some scale $\sim k^{-1}$ which leaves the horizon before the end of the generating phase of perturbations, see Ref. [16], where $k$ is the comoving wave number, and the subscript ' $e$ ' denotes the end time of the generating phase of perturbations, thus $k_{e}$ is the last mode to be generated, and $h \equiv \dot{a} / a$ is the Hubble parameter, where the dot denotes the derivative with respect to the cosmic time. When taking $a h=a_{0} h_{0}$, where the subscript ' 0 ' denotes the present time, we will obtain the efolding number required by observable cosmology. In this case, Eq. (1) is actually the ratio of the physical wavelength corresponding to the present observable scale to that at the end of the generating phase of perturbations.

The evolution of scale factor in the expanding background can be simply taken as $a(t) \sim t^{n},(t \rightarrow \infty)$ and $a(t) \sim(-t)^{n},\left(t \rightarrow 0_{-}\right)$. We will assume that $n$ is a constant for simplicity. In the conformal time $\eta$, we obtain 
$a(\eta) \sim(-\eta)^{n /(1-n)}$. Thus we have

$$
a \sim\left(\frac{n}{(n-1) a h}\right)^{\frac{n}{1-n}} .
$$

To produce the efolding number, i.e. $\mathcal{N}>0$, ah must increase with time, i.e. $\ddot{a}>0$. This suggests that $n>1$ for $a(t) \sim t^{n}$, which corresponds to the accelerated expanding phases, in which $\dot{h}<0$, and $n<0$ for $a(t) \sim(-t)^{n}$, which corresponds to the superaccelerated expanding phases, in which $\dot{h}>0$, e.g. see Ref. [15] ${ }^{1}$. Taking the logarithm in both sides of (2), we obtain

$$
\ln \left(\frac{1}{a h}\right)=\left(\frac{1-n}{n}\right) \ln a .
$$

This equation can be also applied to the expansion with arbitrary constant $n$. We plot Fig.1, in which and also in the whole note we have assumed that after the generating phases of primordial perturbations ends, the "reheating" will rapidly occur and then bring the universe back to the usual FRW evolution ${ }^{2}$. Here the reheating means a transition that the fields or fluids dominating the background decay into radiation.

We can see in Fig.1 that in principle one can not obtain the primordial perturbations in the decelerated expanding phases in which $0<n<1$, however, introducing the modified dispersion relation can change this point. The usual dispersion relation may be generally expected to receive some corrections with the increase of energy, which in some sense can be regarded as a phenomenological description of high energy new physics, see Ref. [19, 20]. These modifications has been applied to the early universe, especially the inflation cosmology, in which the modified dispersion relation can significantly affect the spectrum of primordial perturbations generated during inflation 22, 23]. The modified dispersion relation can also naturally arise from generally covariant scalar field 21]. Following Ref. [14, in the conformal time, one can introduce a projector $h_{\mu \nu}$, which projects onto the space orthogonal to a timelike vector $u_{\mu}=(1 / a, 0,0,0)$ and satisfies $h_{\mu \nu} h_{\rho}^{\nu}=h_{\mu \rho}$ and $h_{\mu \nu} u^{\nu}=0$. Thus one may write $h_{\mu \nu}$ as $h_{\mu \nu}=a^{2} \cdot(0,1,1,1)$. By the help of the projection tensor, we can define a spatial derivative as $\mathcal{D}_{\mu}=h_{\mu}^{\nu} \nabla_{\nu}$, which is orthogonal to $u_{\mu}$ and so has only spatial components, and a time derivative $\left(\delta_{\mu}^{\nu}-h_{\mu}^{\nu}\right) \nabla_{\nu}$. In principle, by combining these two generally covariant derivatives, one can obtain any combination of time and spatial derivatives, so any dispersion relation when acting on a scalar field. For example, if the spatial component of scalar field Lagrangian is as follows $\sim \varphi\left(\mathcal{D}_{\mu} \mathcal{D}^{\mu}\right)^{q} \varphi / a^{p}$,

\footnotetext{
1 The limit case of $n \simeq 0_{-}$can be very interesting [6] and was studied in detail in the island universe model [17, 18].

2 The superaccelerated phase will generally evolve to a Big Rip at late time, unless there is not an exit mechanism or "reheating".
}

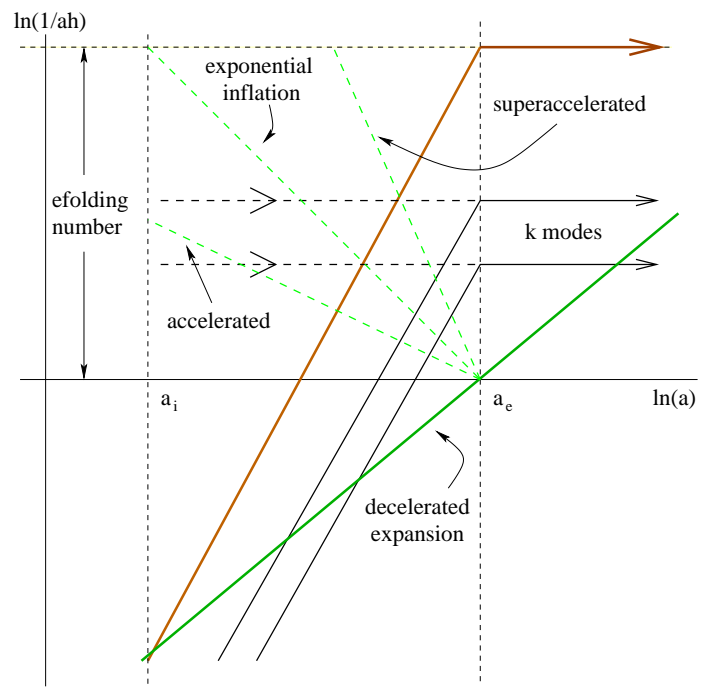

FIG. 1: The sketch of evolution of $\ln (1 / a h)$ with respect to the scale factor $\ln a$ during the generation of perturbations. The left side of $a_{e}$ is the generating phase of perturbations, in which in the region above the $\ln a$ axis the green dashed line corresponding the usual exponential inflation with $|n| \rightarrow \infty$ divides this region into the superaccelerated phase (up right) in which $n<0$ and the accelerated phase (lower left) in which $n>1$. The perturbation modes (black dashed lines) with the wave number $k$ can leave the Hubble horizon during their generations and then reenter the horizon during the radiation/matter domination at late time. In principle it is hardly possible to obtain the causal primordial perturbations in a decelerated expanding phases in which $0<n<1$, see the region below the $\ln a$ axis, since there are nothing leaving the horizon during their evolutions. However, when we introduce the scalar field with the modified dispersion relation $\omega=k / a^{p}$, the similar case to inflation can be imitated in a decelerated expanding phases. In this case the comoving wave number of perturbations will not unchange any more. This makes the evolution of their physical wavelengths able to be faster than that of $1 / h$ when the condition $n(p+1)-1>0$ is satisfied, see the black line and green line.

where $p$ and $q$ are constant, we will have the dispersion relation

$$
\omega=\frac{k^{q}}{a^{p}} .
$$

Here for our purpose we will not pay more attentions to the relevant discussions on the modified dispersion relation. We in the following will focus on the primordial perturbations of the scalar field with the dispersion relation (41) in a decelerated expanding background.

We firstly begin with a simple modification as follows $\omega=k / a^{p}$, where $p$ is the constant, and when $p=0$, it recovers to the normal one. Note also that this case in some sense is similar to that of the fluid with the decaying sound speed [24, 25]. To make a matching to the observable cosmology, it is convenient to define an equivalent "efolding number". Note that with above modification to the dispersion relation, the effective comoving wave 
number $\omega$ will not unchange any more during its evolution and has an extra suppress leaded by $a^{p}$, and thus an increasing $\sim a^{p}$ of the effective comoving wavelength, which directly effects the physical wavelength of correspondent mode. What the efolding number required by observable cosmology actually reflects is the ratio of the physical wavelength corresponding to the present observable scale to that at the end of the generating phase of perturbations, thus the change of physical wavelength induced by the shift of the comoving wave number must be included in the definition of the efolding number. Thus similar to Eq.(11), the equivalent efolding number can be written as

$$
e^{\mathcal{N}} \equiv\left(\frac{a_{e}}{a}\right)^{p} \cdot\left(\frac{k_{e}}{k}\right)
$$

From Eq.(2), we have $\ln \left(k_{e} / k\right)=(n-1) \ln \left(h / h_{e}\right)$. Thus substituting it and Eq.(2) into Eq.(5), we can obtain $\mathcal{N}=(n p+n-1) \ln \left(h / h_{e}\right)$. For an expanding universe with $0<n<1$, we can generally have $h>h_{e}$. Thus to make $\mathcal{N}>0$, which is required by the generation of primordial perturbations, $n(p+1)-1>0$ must be satisfied. This can be reduced to $n>1$ for $p=0$, which corresponds to the usual inflationary cases. In the expanding process, for the field with the normal dispersion relation, the physical wavelength of its modes $\sim a$, and only when the evolution of $a$ is faster than that of $1 / h$, can the primordial perturbations be generated, which can only be implemented in the cases of $n>1$ and $n<0$ (here $h<h_{e}$ ). However, for the field with the modified dispersion relation $k / a^{p}$, the physical wavelength of correspondent modes is $\sim a \cdot a^{p}$, thus even if for $0<n<1$ it is also possible that the evolution of physical wavelength is faster than that of $1 / h$, which leads that the correspondent modes can leave the horizon and thus the generation of primordial perturbation, see Fig. 1 for an illustration. In fact the condition $n(p+1)-1>0$ means that in Fig.1 the lines of $k$ modes must be intersected with that of $\ln (1 / a h)$, i.e. the green line, in the past. If $n(p+1)-1 \simeq 0$, the intersection will be expected to be in infinite far position of lower left side of Fig.1, and in this case the scale of $h$ will be required to be very high. Note that $h$ can be taken to Planck scale at most and in principle $h_{e}$ has also a lower limit, thus generally $n(p+1)-1$ should be far away from 0 in order to obtain enough efolding number.

\section{CALCULATIONS OF SPECTRUM}

In this section we will calculate the primordial perturbations spectrum of scalar field with modified dispersion relation. We assume that this scalar field $\varphi$ dose not affect the evolution of the background. In the momentum space, the motion equation of $\varphi$ is given by

$$
u_{k}^{\prime \prime}+\left(\omega^{2}-f(\eta)\right) u_{k}=0,
$$

where $u_{k}$ is related to the perturbation of $\varphi$ by $u_{k} \equiv$ $a \varphi_{k}$ and the prime denotes the derivative with respect to $\eta$, and $\omega$ is given by Eq.(4) with $q=1$, and generally since $\omega=k / a^{p} \sim k /(-\eta)^{\frac{n}{1-n}}$, which is different from the usual case with $\omega=k$ constant, thus by using the mathematics handbook about deformed Bessel equation, $f(\eta)$ is required to be written as

$$
f(\eta) \equiv \frac{v^{2} r^{2}-1 / 4}{\eta^{2}}
$$

where $v$ is generally required to be nearly constant so that Eq.(6) is solvable, and is determined by the evolution of background and the details of $\varphi$ field, such as its mass, its coupling to the background, and $r$ is determined by the behavior of $\omega(\eta)$. Conventionally, the dispersion relation of scalar field is $\omega=k$, which corresponds to $q=1$ and $p=0$, and thus $r=1$. When $p \neq 0$, we have

$$
r \equiv \frac{n(p+1)-1}{n-1}
$$

The general solutions of this equation are the Hankel functions with the order $v$ and the variable $\omega \eta$. In the regime $\omega \eta \gg 1$, the modes can be regarded as adiabatic. The reason is that note that $\omega^{\prime} / \omega \sim 1 / \eta$, thus the adiabatic condition $\omega^{\prime} / \omega^{2} \ll 1$ is equivalent to $\omega \eta \gg 1$. Note also that $\eta \sim 1 /(a h)$, we have $\omega \eta \sim \omega /(a h) \gg 1$, and thus obtain $a \omega^{-1} \ll 1 / h$, which corresponds to the case that the effective physical wavelength is very deep into the horizon. Thus in this regime we may take

$$
u_{k} \simeq \frac{1}{\sqrt{2 \omega(k, \eta)}} \exp \left(-i \int^{\eta} \omega(k, \eta) d \eta\right)
$$

as an approximate solution of Eq.(6), which in some sense is similar to the case in which the initial condition can be taken as usual Minkowski vacuum. Note that $\omega \eta$ will decrease with the expansion. Thus at late time, we can expect $\omega \eta \ll 1$, i.e. $a \omega^{-1} \gg 1 / h$. The expansion of Hankel functions to the leading term of $\omega \eta$ gives

$$
k^{3 / 2}\left|\varphi_{k}\right| \sim k^{3 / 2-v},
$$

where the other factors without $k$ have been neglected. Thus to obtain the scale invariant spectrum, $v=3 / 2$ is required.

\section{A. massless case}

For the massless scalar field, we have $f(\eta) \equiv a^{\prime \prime} / a$. Note that $a \sim \eta^{n /(1-n)}$, and then use Eqs.(7) and (8), we can obtain

$$
v=\frac{1}{2}\left|\frac{3 n-1}{n(p+1)-1}\right| .
$$

If $p=0$, Eq. (11) will be reduced to the normal case, in which when $|n| \rightarrow \infty$ we can obtain $v=3 / 2$ and 
thus a scale invariant spectrum, which is familiar result in the inflation. From above discussions we have known that $n(p+1)-1$ is require to be larger than 0 for the generation of primordial perturbations. Thus in Eq.(11) taking $v=3 / 2$ required by the scale invariant spectrum, we have

$$
n(p+1)-1=\left|n-\frac{1}{3}\right| .
$$

Substituting it to $\mathcal{N}$ leaded by Eq.(15), we obtain

$$
\mathcal{N}=\left|n-\frac{1}{3}\right| \ln \left(\frac{h_{i}}{h_{e}}\right),
$$

where the subscript ' $i$ ' denotes the beginning time of the generating phase of perturbations. Note that in Eq. (13), the efolding number is not related with $p$, which is also actually valid for the case with arbitrary spectrum, though we are constrained to that with the scale invariant spectrum and then obtain Eq. (13). Thus since $0<n<1$, we see that to obtain enough efolding number required by observable cosmology, the ratio $h_{i}$ to $h_{e}$ must be large enough. Note that $h_{i}$ has an upper limit, i.e. the Planck scale, thus it seems that $h_{e}$ must be taken very low.

The efolding number required is generally determined by the evolution after reheating. In principle the lower energy density is when the generating phase of perturbations is over, the smaller the efolding number required is. For an idealistic case, in which after the generating phases of perturbations ends the universe will rapidly be linked to an usual FRW evolution, one can have $\mathcal{N} \simeq 68.5+(1 / 2) \ln \left(h_{e} / m_{p}\right)[26]$. Instituting it into (13), we can cancel $\mathcal{N}$ and obtain a relation between $h_{e}$ and $n$. We plot Fig.2 in which for various $n$ in the region $0.4<n<1$, the $\log \left(h_{i} / h_{e}\right)$ required are given. We can see that when taking the initial energy scale as Planck scale and the end scale as nucleosynthesis scale, in which we have $h_{i} / h_{e} \sim 10^{40}$, in order to obtain enough efolding number, $n>0.6$ is required, while when the end scale lies in Tev scale, we have $n>0.8$. We may also consider slightly red spectrum i.e. $v>3 / 2$, as was favored mildly by WMAP [1]. From Eqs.(5) and (11), we see that the value of $n$ will be required to be larger. These results indicate that generally it seems slightly difficult to satisfy simultaneously the conditions required by the enough efolding number and the scale invariant spectrum, since the $h_{e}$ required must be very low and in the meantime $n$ is generally constrained in a cabined region. This makes some familiar phases, e.g. the radiation phase and the matter phase, hardly be included in possible applications.

We may introduce the more general dispersion relation, e.g. change the power of $k$, as in Eq.(4) with $q \neq 1$. However, it seems not helpful to relax the above difficult, since this only equals to bring a factor proportional to $1 / q$ in $\mathcal{N}$ obtained from Eq.(5) and the denominator of Eq.(11) simultaneously. They will be generally set off in the calculations obtaining the Eq. (13). Thus to solve the above problem, we must assure that the modifications

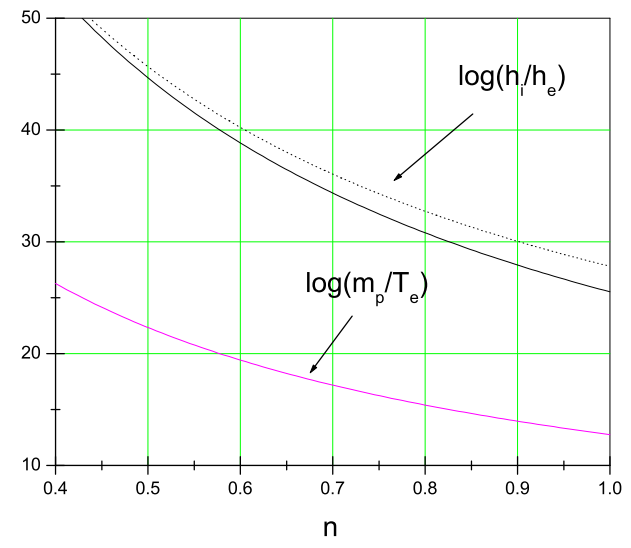

FIG. 2: The value of the $\log \left(h_{i} / h_{e}\right)$ with respect to $n$ in order to obtain enough efolding number. The horizonal axis is $n$. The solid line is the case of $h_{i} \sim m_{p}$ and the dashed line is that of $h_{i} \sim 10^{-4} m_{p}$. The region above the corresponding line is that with enough efolding number. The red line corresponds to the maximal reheating temperature at $h_{e}$ epoch where $h_{i}$ is taken as Planck scale.

introduced dose not change $\mathcal{N}$ obtained from Eq.(5) and the denominator of Eq.(11) simultaneously.

\section{B. massive case}

When the scalar field is massive, $f(\eta)$ is given by $f(\eta)=\frac{a^{\prime \prime}}{a}-\mu(a h)^{2}$, where $\mu \equiv \frac{m_{\varphi}^{2}}{h^{2}}$ has been defined, see Ref. 27] for details on the spectrum of massive scalar field with normal dispersion relation. Note that $a \sim \eta^{n /(1-n)}$, thus we can obtain $f(\eta)$, where $a h(n-1) / n=\eta$ has been used. Note that $h \sim 1 / t$, i.e. it generally changes with the time in the decelerated expanding phase, thus $\mu$ is generally not constant and so the numerator of $f(\eta)$, which will make us very difficult to obtain the analytic solution of Eq.(6). Thus we need to fix $\mu$ constant, which can be done by introducing a non-minimally coupling $\sim R \varphi^{2}$ between $\varphi$ and gravity, where $R \sim h^{2}$ is the Ricci curvature scalar. In this case, we will have that $m_{\varphi}^{2} \sim R \sim h^{2}$, and so $\mu$ can be a constant. Thus Eq.(6) becomes solvable exactly. From $f(\eta)$, and then using Eqs.(7) and (8), we can obtain

$$
v_{m}^{2}=v^{2}-\left(\frac{n}{n(p+1)-1}\right)^{2} \cdot \mu
$$

which is used to replace $v$ in Eq.(10), where the subscript $m$ denotes the value of $v$ for the massive scalar field. The scale invariance of spectrum requires $v_{m}=3 / 2$, thus with 
Eq.(14), we can obtain

$$
n(p+1)-1=\sqrt{\left(n-\frac{1}{3}\right)^{2}-\left(\frac{2 n}{3}\right)^{2} \cdot \mu} .
$$

Note that the term inside sqrt in Eq.(15) should be larger than 0 , which suggests $\mu<(3 / 2-1 /(2 n))^{2}$. For $0<n<1$, the range of $(3 / 2-1 /(2 n))^{2}$ lies between 0 and $\infty$. Thus to obtain enough efolding number, $\mu<0$ is generally required, which corresponds to introduce a scalar field with negative mass term. Substituting Eq.(14) into $\mathcal{N}$ obtained from Eq.(5), we can obtain

$$
\mathcal{N}=\left(\sqrt{\left(n-\frac{1}{3}\right)^{2}-\left(\frac{2 n}{3}\right)^{2} \cdot \mu}\right) \ln \left(\frac{h_{i}}{h_{e}}\right) .
$$

Thus one can see that to satisfy the requirements of observable cosmology, for the decelerated expanding phase with $0<n<1$, the enough efolding number may be obtained by properly selecting the value of $\mu$ in the case with fixed $n$ in Eq.(16), while when $n$ and $\mu$ are fixed by Eq.(16), the scale invariant spectrum may be obtain by properly matching the value of $p$ in Eq.(14). We plot Fig. 3 , in which for arbitrary $n$ in the region $0.4<n<1$, in order to obtain enough efolding number, the $-\mu$ required with respect to $n$ is given. We can see that for different value of $n,-\mu$ lies in an acceptable region and in the meantime $h_{i} / h_{e}$ is not required to be very large, which is from the case with the massless scalar field. Note that both cases in Fig.3 can not be implemented in the massless field, in which the value of $h_{i} / h_{e}$ in Fig.3 is not so large to ensure enough efolding number.

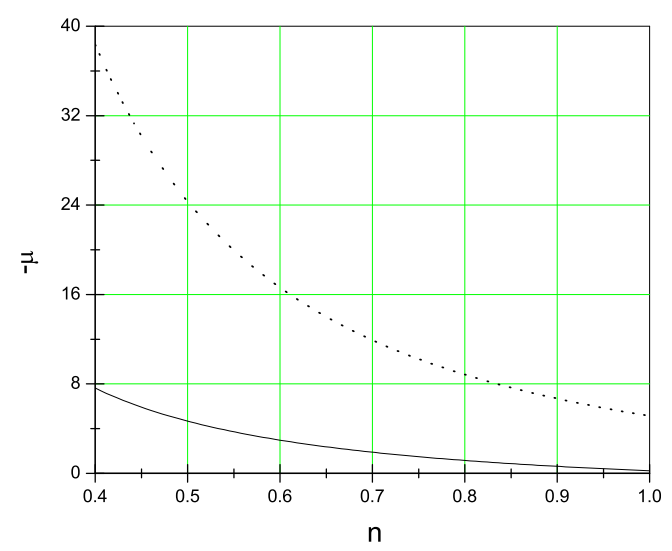

FIG. 3: The value of $-\mu$ with respect to $n$ in order to obtain enough efolding number. The vertical axis is $-\mu$ and the horizonal axis is $n$. The solid line corresponds to the case of $h_{i} \sim 10^{-4} m_{p}$ and $h_{e} \sim \mathrm{Tev}$, and the dashed line is that of $h_{i} \sim m_{p}$ and $h_{e} \sim 10^{-9} m_{p}$.

\section{SUMMARY AND DISCUSSION}

The modification of the dispersion relation of the scalar field brings a possibility generating the primordial perturbations in a decelerated expanding background, however, we find that in order to generate a nearly scale invariant spectrum, it will be slightly difficult to obtain enough efolding number required by the observable cosmology in a simple case. But when we consider more general cases, e.g. the massive scalar field, the problem can be relaxed. Thus though the conditions required look like slightly special, it seems possible to seed the nearly scale invariant primordial perturbations within a conventional evolution not involving the inflation. These perturbations may be transferred to the curvature perturbations at late time by some mechanisms, e.g. as in Refs. [28, 29], thus may be interesting and responsible for the structure formation of observable universe. Note that current observations actually favor a red tilt spectrum [1], but not exact scale invariant one. However, this does not pose any problem here, since we can always set any value of $p$ in Eq.(11) or (14) to obtain the $v$ or $v_{m}$ required by the red tilt of spectrum, even the blue tilt. In principle there is not the generation of primordial gravitational wave, since the seeding of scalar perturbation occurs during a decelerated expansion, unless the sound speed of the gravitational wave is also time dependent, as in the case of scalar perturbation. Non-Gaussianity is expected to be small. However, we still need to a detailed discussion on the gravitational wave and non-Gaussianity in order to match the coming observable tests. In addition, since the energy scale when the generating phase of primordial perturbations ends may be very low to Tev, even BBN scale, it is also interesting to study whether there are some observable effect on e.g. baryogenesis. Thus it seems that many significant issues related to this work remain. We expect to back to these studies in the future.

Finally, it should be pointed out that such a decelerated evolution of early universe can not solve all problems of standard cosmology, as has been explained in the inflation models. For example, here initially the homogeneity in the superHubble scale must be imposed. Be that as it may, however, this work displays an "unnatural" but possible example seeding a phenomenologically realistic spectrum of primordial perturbations in a nonaccelerated expanding background, which to some extent highlights the fact again that identifying the origin of primordial perturbations may be a much more subtle task than expected.

Acknowledgments The author would like to thank C. Armendariz-Picon for discussions. This work is supported in part by NNSFC under Grant Nos: 10405029, in part by the Scientific Research Fund of GUCAS(NO.055101BM03), as well as in part by CAS under Grant No: KJCX3-SYW-N2. 
[1] D.N. Spergel et.al. astro-ph/0603449.

[2] A.H. Guth, Phys. Rev. D23 (1981) 347.

[3] A.D. Linde, Phys. Lett. B108 (1982) 389; A.A. Albrecht and P.J. Steinhardt, Phys. Rev. Lett. 48 (1982) 1220.

[4] J.M. Bardeen, P.J. Steinhardt and M.S. Turner, Phys. Rev. D28 679 (1983).

[5] V. Mukhanov and G. Chibisov, JETP 33549 (1981); A.H. Guth and S.-Y. Pi, Phys. Rev. Lett. 49, 1110 (1982); S.W. Hawking, Phys. Lett. B115,295 (1982); A.A. starobinsky, Phys. Lett. B117 175 (1982).

[6] Y.S. Piao and E Zhou, Phys. Rev. D68, 083515 (2003).

[7] Y.S. Piao and Y.Z. Zhang, Phys. Rev. D70, 063513 (2004).

[8] P.F. Gonzalez-Diaz and J.A. Jimenez-Madrid, Phys. Lett. B596, 16 (2004).

[9] M. Baldi, F. Finelli and S. Matarrese, astro-ph/0505552.

[10] J.W. Moffat, Int. J. Mod. Phys. D2 351 (1993).

[11] A. Albrecht and J. Magueijo, Phys. Rev. D59, 043516 (1999); J.D. Barrow and J. Magueijo, Phys. Lett. B443, 104 (1998).

[12] G.F.R. Ellis, J.-P. Uzan, Am. J. Phys. 73, 240 (2005).

[13] A. Liddle, Phys. Rev. D51 5347 (1995).

[14] C. Armendariz-Picon, astro-ph/0606168.

[15] Y.S. Piao and Y.Z. Zhang, Phys. Rev. D70, 043516
(2004); Y.S. Piao, Phys. Lett. B606 (2005) 245.

[16] J. Khoury, P.J. Steinhardt and Neil Turok, Phys. Rev. Lett. 91161301 (2003).

[17] Y.S. Piao, Phys. Rev. D72, 103513 (2005);

[18] Y.S. Piao, Phys. Rev. D74, 043509 (2006).

[19] W.G. Unruh, Phys. Rev. D51 2827 (1995).

[20] S. Corley and T. Jacobson, Phys. Rev. D54 1568 (1996).

[21] T. Jacobson and D. Mattingly, Phys. Rev. D63 041502 (2001).

[22] J. Martin and R.H. Brandenberger, Phys. Rev. D63 123501 (2001).

[23] R.H. Brandenberger and J. Martin, Mod. Phys. Lett. A16 999 (2001).

[24] P.P. Avelino and C.J.A.P. Martins, Phys. Rev. Lett. 85 (2000) 1370; Phys. Rev. D62 (2000) 103510.

[25] C. Armendariz-Picon and E.A. Lim, JCAP 0312 (2003) 002 .

[26] A.R Liddle and S.M. Leach, Phys. Rev. D68 103503 (2003).

[27] Y.S. Piao and Y.Z. Zhang, gr-qc/0608058.

[28] G. Dvali, A. Gruzinov and M. Zaldarriaga, Phys. Rev. D 69023505 (2004); Phys. Rev. D 69083505 (2004).

[29] L. Kofman, astro-ph/0303614 